\title{
8th Annual rare disease scientific workshop: Evaluating early access models for patients: Flashpoints, frameworks \& case studies for advancement: A summary
}

Bryan A. Wilson ${ }^{1,2}$, Monte S. Willis ${ }^{1,3^{*}}$

'McAllister Heart Institute, University of North Carolina, Chapel Hill, NC USA

${ }^{2}$ Department of Psychology and Neuroscience, University of North Carolina, Chapel Hill, NC USA

${ }^{3}$ Department of Pharmacology, Pathology \& Laboratory Medicine, University of North Carolina, Chapel Hill, NC USA

Article Info

\section{Article Notes}

Received: February 04, 2016

Accepted: February 07, 2017

\section{*Correspondence:}

Monte S. Willis, University of North Carolina at Chapel Hill McAllister Heart Institute, Department of Pathology \& Laboratory Medicine 103 Mason Farm Road, 2340B Medical Biomolecular Research Building Chapel Hill, NC 27599-7525 USA, Tel: +1 (919) 8431938 / 5630,

E-mail: monte_willis@med.unc.edu

(c) 2017 Monte S. Willis. This article is distributed under the terms of the Creative Commons Attribution 4.0 International License.

\section{Keywords}

Rare disease

Drug development

Expanded access

Conference

\section{ABSTRACT}

The 8th annual Rare Disease Scientific Workshop featured presentations on the topic of "Evaluating Models to Provide Early Access for Rare Disease Patients". The workshop included presentations from and participation by experts in Industry, the Food and Drug Administration, and academia focused on case study examples of successful mechanisms for those seeking to provide early access to experimental therapies for patients. Current regulatory frameworks were discussed and evaluated, with an emphasis on rare and serious diseases with the goal of identifying best practices and useful ideas for a set of standard approaches to early access.

Date: Tuesday, September 13, 2016, Washington, DC

Location: Willard Intercontinental Hotel, Washington DC

Sponsor: The EveryLife Foundation for Rare Diseases

Non-standard abbreviations: AAV, adeno-associated virus; ALS, Amyotrophic Lateral Sclerosis; CURES, Compassionate Use Reform and Enhancements; DMD, Duchenne Muscular Dystrophy; ERT, enzyme replacement therapy; FDA, US Food and Drug Administration; GLPs, good laboratory practices; ICU, intensive care unit; IND, FDA's Investigational New Drug program; H.R., House of Representatives; IRB, institutional review boards; J\&J, Johnson and Johnson; JAMA, Journal of the American Medical Association; MCT, Medium-chain triglyceride; MPS I, mucopolysaccharidosis I; MPS7, Mucopolysaccharidosis type 7; NYU, New York University; OHOP, Office of Hematology and Oncology Products; rhGUS, recombinant human betaglucuronidase; RUF, Reagan-Udall Foundation; WT, WideTrial

\section{Introduction}

Max G. Bronstein, Senior Director of Advocacy and Science Policy, EveryLife Foundation for Rare Diseases

Emil Kakkis, M.D., Ph.D., President, EveryLife Foundation for Rare Diseases \& CEO, Ultragenyx, Inc.

The theme of the 2016 meeting centered around "Evaluating Early Access Models for Patients" and includes presentations from leaders and experts from industry, government, and academia. Organized into five distinct sessions, the workshop provided a current assessment of the barriers faced by patients, physicians and sponsors on identifying, recruiting and maintaining regulatory standards on expanded access programs. Critically, these barriers 
often delay patient access to therapies that may provide benefit for many rare diseases. Speakers were asked to focus heavily on real-world examples of various programs and initiatives currently being developed to tackle this issue. Practical tips were provided for patients with rare diseases, their families, medical providers, and the public to understand and access the emerging expanded access resources.

\section{Session I: Origins, Flashpoints, and Defining Moments for Expanded Access}

Unpaking expanded access to Investigational Drugs and Biologics. Speaker: Richard Klein, Director of Patient Liaison Program, FDA

In the process of developing drugs for rare diseases, it is critical to leverage the recent expanded access pathways provided by the FDA (Federal Drug Administration) for investigational drugs and biologics. The overall goal of expanded access is finding treatment where there currently is none. The traditional FDA drug approval process is the most effective way to ensure patients have therapies while maintaining patient safety. Traditional FDA approvals are based on rigorous clinical trials and quality assurance assessments to predict and mitigate potential risks. Patients with life-threatening rare diseases cannot afford to wait until drugs are approved. Standard treatment options for patients with rare disease are often not available or quickly exhausted, resulting in no effective therapies for their conditions. Thus, expanded access allows patients to gain access to unapproved or investigational therapies that may provide clinical benefit.

While the historical inception of utilizing expanded access for multiple diseases dates back to the 1960's and 1970's, no official regulatory mandates were established until 1987 when investigational new drug (IND) regulations were revised to provide access for broader patient populations (Treatment IND/Protocol - 21 CFR 312.24). These regulations were further revised in 2009 (21 CFR 312/ IND Regulations). This included the consolidation of treatment use into a separate subpart of the IND regulations containing all necessary information in one place (Subpart I) and described three distinct categories for expanded access: 1) Individual, 2) Intermediate size population, 3) Treatment IND. Individual patient expanded access programs are often physician initiated with them serving in a dual role as sponsor/investigator. The physician is responsible for all sponsor activities including study tracking, reporting, and alternative event monitoring. Intermediate size population expanded access programs are intended for patient populations smaller than intended for treatment IND ( 100 patients) and may include physicians, manufacturers or $3^{\text {rd }}$ parties as sponsors. Sufficient evidence of the drug's safety at the proposed dose and duration is needed to justify the size of the population to be exposed. Moreover, preliminary evidence (clinical and pharmacological in nature) should be provided. Treatment IND expanded access programs include drugs that are being investigated in clinical trials designed to support marketing, or trials are already completed. Typically, companies are actively pursuing marketing approval and evidence of safety and effectiveness as evidenced from phase II or phase III clinical trials.

A framework for implementing expanded access programs. Consider the implementation of expanded access a community responsibility that includes patients, physicians, industry sponsors, the FDA and institutional review boards (IRBs) that are responsible locally for the ethical considerations of treating patients. The FDA website has resources to assist individuals in applying for the various categories of expanded access. In addition, two notices released by the FDA in the last year may be helpful: 1) Expanded Access to Investigational Drugs for Treatment Use - Questions and Answers (Guidance for Industry) (See https://goo.gl/9p6m1G); and 2) Charging for Investigational Drugs Under an IND - Questions and Answers (Guidance for Industry) (see https://goo.gl/ ldjWdb).

Expanded Access Programs for Drugs and Biologics. Speaker: Richard A. Moscicki, M.D., Deputy Center Director for Science Operations, Center for Drug Evaluation and Research, FDA

The barriers to the expanded access programs for drugs and biologics faced by patients looking for access to new drugs can be grouped into two main categories: 1) Physician and patient knowledge of the process both in terms of interfacing with regulatory agencies and sponsors; and 2) Concern that expanded access might adversely affect the clinical development process and the regulatory review of drug clinical trials. It is important to understand if adverse effect data from expanded access use has a negative impact on future regulatory/developmental mandates.

Adverse events rarely are not commonly the cause of discontinuing a drug and are expected in patients with disease. Adverse events are expected in general patient populations due to the underlying disease itself (including rare diseases). There are currently no special requirements for expediting the reporting of adverse effects for standard access. The requirement for standard IND applications is to report serious, unexpected adverse reactions. However, in the context of standard access, patients are severely ill and not all adverse reactions are equally damaging to the reputation of the drug being tested.

To evaluate the influence of reporting adverse effects, the FDA analyzed their tracking system over a ten-year 
period (2005-2015). Within this data set, a total of 10,597 INDs were evaluated 360 commercial INDs and 665 standard access INDs submitted to the sponsors for commercial INDs. Out of over 10,000 INDs examined, only two instances of patient death, shortly after administration of an investigated drug were reported within expanded access programs resulting in clinical holds $0.2 \%$ incidence). Despite this, the clinical holds were indefinite and were resolved within 2-3 months, thus allowing the continuation of the programs.

A small number of programs are rejected in the clinical trials process, but the focus is on safety, not adverse events. If patient safety from drug effects is found independent of adverse events, programs are discontinued to guard patient safety. Due to the complex regulatory processes surrounding standard access program implementation and monitoring, the FDA is partnering with the ReaganUdall Foundation to establish a national navigator to assist people with these processes. The navigator serves as a clearinghouse providing information, education and support to standard access. Overall, it will assist the patient and physician communities about potential sponsors for expanded access to expedite the process by which patients are exposed to treatment options.

\section{Session II: Navigating the World of Expanded} Access

Navigating Expanded Access. Speaker: Elena Gerasimov, Director, Compassionate Use Navigator Program, Kids v Cancer, Washington, DC

The Kids v Cancer (a non-profit advocacy group for pediatric research) recently conducted a survey to better understand the need and utilization of expanded access programs. Of the 74 pediatric oncologists surveyed in New York, 59 supported the idea of providing a service to assist patients and physicians in navigating the informational barriers towards preparing standard access applications (i.e.single patientIND).Thirty-nine(39) physicians reported they had never applied for compassionate use themselves, with 8 not familiar with concept of compassionate use itself. Out of the 25 physicians reporting their prior experience applying for an investigational drug's compassionate use, 17 "sometimes" observed clinical benefit ( $50 \%$ of cases), while 7 "often" observed clinical benefit ( $\sim 80 \%$ of cases). While the study is on-going, these findings support the need for the development of resources to assist physicians on multiple levels. The Kids v Cancer "Compassionate Use Navigator" was created to help physicians treating patients needing compassionate use access to treatments.

The Compssionate Use Navigator (https://goo.gl/ GOa6jq) functions as a website, database, and personal assistant service for expanded access programs. It provides points of contact for patients and physicians to expanded access. Patients and physicians can gain access to key points of contact and potential drug companies who may be interested in sponsoring an investigational therapeutic. Moreover, the navigator provides templates and forms for IRB applications for unapproved drugs, as well as sample query letters to drug companies.

Ancillary to its primary focus of assisting patients and families, the navigator is also building a registry of requests and clinical outcomes of expanded access programs; these can be either self-reported by a family or a physician. Initially focusing on cancer, Gerasimov highlighted information from the FDA's Office of Hematology and Oncology Products (OHOP) as an example of the type of data Kids v Cancer envisions constructing with their patient navigator. OHOP data on expanded access requests for pediatric oncology patients from January 2012-December 2014 reported 1,332 INDs (single patient and emergency use applications). Requests were for 157 unique drugs to treat a variety of cancer-related diseases including Chronic Myeloid Leukemia (28\%), breast cancer (12\%), Acute Lymphoblastic Leukemia (9\%). Age data was available for 827 patients ( $8 \%$ age 17 or younger), however data on efficacy and toxicity was limited.

A more comprehensive understanding of whether some investigational drugs were effective or caused toxicity, is still needed. Transparency is critical in all stages of drug development including expanded access pipelines, as recently outlined in the Journal of the American Medical Association (JAMA) article, "FDA Expanded Access Programs for Experimental Medicines" (PDF reprint available for download at: https://goo.gl/oMvVHO). Increasing transparency addresses the unfair advantages people and/or physicians at large comprehensive cancer centers have with accessing expanded access programs by providing their experiences to everyone. In the next year, Kids v Cancer will enhance their database by increasing its educational offerings to physicians and families to assist them in finding investigational drugs for potential use, while navigating the regulatory processes of applying for IRBs and expanded access.

Exploring an Expanded Access “Navigator". Speaker: Nancy Beck, Ph.D., Acting Deputy Director, Reagan-Udall Foundation (for the Food and Drug Administration)

The Reagan-Udall Foundation (RUF) is a nonprofit organization established by Congress to help advance the mission of the FDA. Broadly, the RUF creates private partnerships to leverage external resources, expertise, funding, and data to address and solve problems in designing and conducting regulatory science research. This includes the patient-centered expanded access database Navigator. In contrast to the Kids v Cancer patient navigator, the Navigator provides information on a diversity of patient populations and diseases, beyond pediatric cancers. 
The Navigator Interface was created to assist physicians outside of academic medical centers with understanding the process of applying for expanded access, since they may not be aware of the resources available to patients or have the IRB infrastructure readily accessible. The RUF is examining the expanded access landscape and deciphering which gaps still need to be filled. For example, recently stakeholder surveys at workshops and open solicitations of the public, revealed that the access to data on the administration and utilization of expanded access is quite limited. Similarly, very few drug companies who could serve as potential sponsors of investigational therapies, have website or marketing materials detailing their potential interest in expanded access programs.

To address these problems, RUF's strategy is to provide healthcare provider education on expanded access as well as establishing a directory of company information who may be willing to sponsor investigational therapeutics. The intended outcomes of this project would be to increase the understanding amongst healthcare providers of the expanded access process and the steps needed to apply. Ancillary to assisting physicians, the RUF envisions their compassionate use navigator as a tool to increase health equity, as both patients and healthcare providers alike will be made aware of resources.

The RUF compassionate use navigator will model itself to provide vital information on drugs that are currently difficult/impossible to access. The 21st Century Compassionate Use Reform and Enhancements (CURES) Act encourages drug companies to provide four website components on expanded access: 1) a point of contact, 2) eligibility criteria, 3) description of the request process, and 4) the anticipated turnaround time. Among 29 drug company websites RUF recently searched to identify this information, 0 of 7 small companies provided this information, and only $18 \%$ of the larger companies provided all four pieces of information. Sixty (60) percent of the larger companies provided at least one of the four pieces, while $22 \%$ provided none. Among the companies that did provide this information, RUF identified considerable variety in the depth and clarity of the information. The RUF wil continue its efforts on honing the functionality of existing expanded access data and determining best practices for educating healthcare providers.

Patient Perspective on Access. Speaker: Eve Bukowski, Vice-President of State Government Relations, California Life Sciences Association \& Cancer Patient

Eve Bukowski, currently battling stage 4 colon cancer, spoke on her experiences as a patient advocate for expanded access in a pre-recorded presentation. In her role as VicePresident of the California Life Sciences Association, she lobbies the California state legislature to support access/ innovation to education in healthcare. She has battled colon cancer for nearly 9 years and is one of a small subset of patients to have survived through two cancer immunotherapy trials. While she was initially unprepared to battle her condition (e.g. 33 surgeries including 2 colon resections), she continues to and is dismantling patient survival statistics in the process. According to the American Cancer Society, the 5-year relative survival rate of metastatic stage 4 colon cancer is $11 \%$. Bukowski considers herself to be in full battle mode and understands that statistics are perspective based, and do not entirely hold true for all patients. In fact, she commended her oncologist for suggesting that she avoid viewing any form of survival statistics related to her disease.

Bukowski remains hopeful that the advances in biomedical research will continue to provide emerging treatment options. She envisions a world where more targeted therapies for cancer are available, with fewer side effects and damage to healthy tissue. Recently, she was enrolled in a new immunotherapy trial and is currently undergoing weekly infusion treatments. Bukowski acknowledged that none of this would be possible if it weren't for the interactions of research institutions, government bodies, funding sources, patient advocacy groups, and medical therapeutic companies, all working together to generate options for patients and restore hope.

Session III: Frameworks \& Models for Expanded Access

A Strategy for Managing Early Access for Orphan Drugs. Speaker: Emil Kakkis, M.D., Ph.D., President, EveryLife Foundation for Rare Diseases \& CEO, Ultragenyx, Inc.

For patients with the rare disorder mucopolysaccharidosis I (MPS I), the navigation of early access programs to experimental drugs has shown some success. While developing the enzyme replacement drug therapy laronidase (Aldurazyme), a Croatian family whose son was suffering from terrible airway problems, storage issues, and liver dysfunction contacted Dr. Kakkis. While preliminary data demonstrated efficacy in relieving some of these medical complications of MPS I, his team was not prepared to test the drug in patients. With no early access plan in place for laronidase and numerous requests from severely infected patients began to come in, they considered four critical issues during the development process: 1) concern of how a patient death might negatively impact the drug's regulatory review and approval process; 2) the costs and distraction from development, 3) concerns about maintaining a drug supply; and 4) FDA not being fully convinced of the drug's benefit after phase II trials.

As patients who did not receive the drug continued to die from MPS I, it became even more apparent that 
further considerations in the crisis of early access for laronidase were needed. However, the issues surrounding the regulatory and development aspects of the drug could not be ignored. Moreover, the question of whether patients should wait until the completion of phase II clinical trials was up for debate, as most patients with MPS I would likely not qualify for these trials or die before they were completed. The dilemma of scientific rigor vs. compassion was challenging. Three three strategies were applied to balance the emerging need for industry to engage in the crisis that exists in the lives of patients: 1) Expanded access (compassionate use); 2) Companion studies for nonqualifying patients; and 3) Investigator-sponsored trials.

Expanded access can be applied following a phase II trial suggesting a drug's efficacy/safety for the same indication in development. Life-threatening/disabling, rapid progression diseases with serious repercussions should be considered for this strategy. A companion study is suggested for patients who do not qualify for phase III trials. These studies are typically smaller and easier to conduct with less cost. Companion studies are good for non-urgent life-threatening disease that progress slowly, allowing for the tailoring of specific study design for these patients. Investigator-sponsored studies should be utilized for indications not in the development program. For example, a company receives several requests for one product in many other disease indications. While no easy answers for the complex issues surrounding early drug access exist, drug companies should continually consider the following aspects: 1) Patient-focused transparency (Website point of contact listed), 2) Prospective process for managing cases (Teams in place to manage requests) and 3) Set of programs in the clinical development portfolio, based on the urgency of disease.

Expanded Access Perspectives. Speaker: Kay Holcombe, Senior Vice-President for Science Policy, Biotechnology Innovation Organization

The measure of a company is not what it can do for hundreds of people but what it can do for one person. In doing so, companies in the biopharmaceutical industry should be dedicated and committed to listening to patients to understanding what patients need and to try and meet those needs by developing safe and effective therapies and bringing them to market. As companies address the needs of patients, they should be aware that some will be interested in expanded access options. They may decide with their healthcare providers they could be helped by taking a specific investigational drug. However, navigating the appropriate strategies to deal with this matter is complex for physicians, especially when patients need access to unapproved investigational drugs before the data on the drug has demonstrated that the drug is safe and most importantly that the drug works.
Companies should consider several points when grappling with the issue of expanded access requests. For instance, if a company knows that their therapy is promising for a specific disease, they should anticipate ways to handle access to the drug before the drug is FDA approved. This is particularly relevant for companies presenting positive drug results at a national or international scientific conference. To prepare for these requests, five (5) items should be considered. 1) How are they going to achieve truly informed consent? When companies can reveal only limited information on the efficacy and safety of an investigational drug (e.g. due to confidentiality agreements), it may be impossible for a company to ask a patient to give informed consent in a transparent manner. 2) How will companies monitor the lack of control they have in a patient's treatment to properly interpret the data? The quality control standards companies have in operating clinical trials are one reason their results can be powerful. When an individual is provided with an investigational compound drug (i.e. investigator-initial trial or single patient IND), these controls are not applied, making the companies knowledge of the drug's context difficult. 3) How are regulators going to evaluate the information that comes from expanded access use, or non-controlled clinical trials use for an investigational drug? Small companies (particularly) worry that the untimely death will trigger the FDA to stop the trial, causing a potential exiting of investigators, decreasing the chances of the drug being further developed. This is a rare worst-case scenario, which only happens 2 out of over 1000 times. 4) How companies provide a scenario to encourage enrollment in their standard clinical trials? People who enroll in standard clinical trials are randomized to receive the experimental medicine or placebo as the standard of care. When patients and physicians find that expanded access is available, they opt out of phase II clinical trials because they don't have a $100 \%$ chance of being administered the actual investigational drug compound. 5)

How do companies maintain their fairness to patients? Companies worry about fairness for multiple reasons. For example, there may be limited amounts of an investigational compound available in advance of its approval due to manufacturing capabilities and costs. Another reason for this is the fact that drugs cannot be mass produced until FDA approval is granted. Thus, for expanded access trials, companies grapple with deciphering the need for patients on a case by case basis.

The Biotechnology Innovation Organization actively works with the FDA and other regulators to expedite access to approved products. They work with companies to improve their clinical trials process, including the IRB review components. They also support 21 st Century CURES Act and new legislation to change the drug development processes to create tools that make clinical trials more creative, more accessible, and faster. 
Expanded Access. Speaker: Sascha Haverfield, D.Phil, Senior Vice-President for Scientific and Regulatory Affairs, PhRMA

The shared goals of patients, healthcare providers, and companies in expanded access is to provide safe and effective medicines available to all patients (meaning the medications are FDA approved). However, the FDA approval process can be lengthy (10-14 years), with very few drugs (1 in 10,000) making it through the pipeline to become FDA approved. There is a continuous need to assess ways of modernizing the drug discovery pathway and how organizations can work with the FDA to streamline the regulatory system to accelerate the drug availability in the United States.

Another shared goal related to expanded access is the need to be treating patients on a more individual basis. The most important part of expanded access is facilitating discussion between an individual, their physician, and the drug company to understand the available options. For many rare disorders, patients have exhausted their possibilities of ever entering standard clinical trials, leaving expanded access as their last option for consideration.

In the last 3.5 years, PhRMA has established principles on expanded access to investigational drugs (https://goo.gl/ F0308p):1)Establishtelephoneorinternet-basedinformation sources to facilitate communication about expanded access programs; 2) Identify if a patient has a serious or lifethreatening illness; 3) Determine if investigational drugs should be under active clinical development; 4) Identify if a patient is ineligible for, or otherwise unable to participate in, clinical trials; and 5) Weigh the potential benefit to the patient with the potential risks.

Summary. The FDA oversight of investigational medicines is essential to ensure patients receive medicines that are safe and effective. At the same time, patients and healthcare providers need better access to accurate and clear information on expanded access programs, including a way to navigate the expanded access process and public dissemination of biopharmaceutical companies' expanded access policies, including request procedures, evaluation criteria, and response timeframes. The timeframe for FDA action on expanded access requests could be considered, and clear FDA guidance on the use of safety and efficacy data derived from expanded access cases is needed. Consideration of the use of real-world evidence (RWE) for regulatory decision-making and assessment of whether institutional review board review of individual patient expanded access requests is necessary to protect patients should also be considered.

Evaluating Early Access Models for Patients...A Business Solution for Better Clinical Drug Development in Serious Unsolved Diseases. Speaker: Jess Rabourn, CFA, Managing Director, WideTrial Expanded Access Clinical Trials and Ax-S Pharma.
WideTrial (WT) is a patient-centered platform launched in 2012 created to lift the burden of expanded access off the shoulders of commercial drug development by introducing it to the nonprofit space. As a central platform, WT acts as an independent nonprofit organization working on behalf of the medical community, patients, and community foundations to partner with drug companies. WT originally established itself as a leader in the expanded access clinical trial space by targeting neurodegenerative diseases through the facilitation of the Amyotrophic Lateral Sclerosis (ALS) emergency treatment fund.

WideTrial consists of four components designed to maintain a centralized nonprofit platform that can be a guiding tool for anybody interested in pursuing expanded access and meeting an unmet need. Component 1) A central nonprofit platform that can be a guiding tool for anyone interested in pursuing expanded access and meeting an unmet need. As a nonprofit party, WT alleviates some of the public relations and political pressure a drug company may be facing if they are worried about early sales before a drug is fully developed. Component 2) Charitable resources. WT serves as a bridge for charitable money. Component 3) Positioned as a partner who can leverage the access to each disease community. Research centers and service providers. Component 4) Leveraging secondary data assets that stem from extended trials or WT (companion). This may be used as a discovery tool to generate strategies of shifting hard costs and reducing public relations and regulatory risks management.

Since real-world data is important to the expanded access process, WT will integrate well-designed and preplanned data-generating access trials into the drug development process primarily by better reporting of patients who are responding to treatments. With this information, WT foresees the development of molecular level fingerprints to better inform companies and assist them in enriching their trial design. As an example, after completing phase II trials, companies may consider including ineligible participants into an extension study, allowing the further examination of the molecular traits of the patients that respond and identify additional parameters within the clinical development process.

\section{Session IV - Industry Case Studies}

Industry Case Study: Expanded Access at Ultragenyx, Hank Mansbach, MD, Vice-President of Medical Affairs, Ultragenyx, Inc

Ultragenyx develops therapies for metabolic genetic disorders, addressing both rare diseases and new drug development programs for these unique disorders. When they are approached with requests for the use of a drug for expanded access, they initially assess whether their portfolio of drugs are suitable to treat the disease they are 
getting a request for. They also determine if alternative options have been explored, such as established on-going clinical trials? They also consider the benefits and risks involved to come to a final decision which is always a challenge.

Example 1: Mucopolysaccharidosis type 7 (MPS7). Mucopolysaccharidosis is an extremely rare disease impacting $\sim 200$ patients worldwide caused by a mutation in the $\beta$-glucuronidase enzyme. The resulting $\beta$-glucuronidase deficiency leads to the inability to process complex sugars, causing the buildup of carbohydrates throughout the body. People with MPS7 have manifestations in the heart, lung, liver, and can present with skeletal abnormalities. MPS7 is considered a spectrum disease, spanning from a severe infantile form and several attenuated forms. While often fatal early in life, MPS7 patients live well into their 20s or 30s. Ultragenyx has developed UX003 (recombinant human beta-glucuronidase, rhGUS) as an investigational therapy for MPS7. Other enzyme replacements developed for nonMPS7 Mucopolysaccharidoses have been developed and led to FDA approved drugs. Currently, UX003 is in an ongoing Phase II clinical trial in the United Kingdom.

In 2013, as Ultragenyx was planning their phase III study for UX003, the family of a 12-year-old patient contacted Ultragenyx to request early access. Their son had become critically ill with acute respiratory failure and was in the intensive care unit (ICU). His healthcare providers contacted Ultragenyx for emergency access as they thought enzyme replacement therapy (ERT) could help. This was an immediate life threating situation with no options for him to participate in a trial. Moreover, no clinical studies had been performed to suggest the drug could work (only pre-clinical animal studies had been performed). Data from a previously developed ERT supported the hypothesis that UX003 might provide some respiratory benefit. So Ultragenyx decided to allow treatment and consulted the FDA. They agreed to grant Matthew early access, and the treatment was given. The patient recovered and continues ERT two years later. His expanded access trial later supported UX003's Phase III study.

Example 2: Long-chain fatty acid oxidation disorders. Defects in long-chain fatty acid oxidation are genetic diseases that result in impaired mitochondrial processing of fatty acids, leading to cellular energy defects. Defects in multiple enzymes lead to this clinical syndrome, which is picked up through newborn screenings in the United States early in life. Like MPS7, Long-chain fatty acid oxidation disorders are spectrum diseases, with some patients living only a few weeks and others living a nearly full life span. Symptoms include cardiomyopathy, hypoglycemia, Rhabdomyolysis, fatigue, and risk of sudden death. One therapy for these diseases is dietary, where fasting is avoided, and patients are supplemented with
Medium-chain triglyceride (MCT) oil. Ultragenyx licensed UX07 (triheptanoin), composed of three seven-carbon fatty acids, to treat long-chain fatty acid oxidation disorders. UX07 was designed to restore mitochondrial metabolism and improve energy production in cells.

A physician used UX07 as compassionate use for his patients and was collecting data without a formal protocol or any end points. Upon learning this, the drug was licensed to conduct formal development trials. To date, the full development of UX07 is underway for adults and older children who are symptomatic but don't have the crisis that the young children do. Ultragenyx is currently working on manufacturing the product. In the interim, the company continues to receive requests for UX07 primarily for infants in crisis with cardiac failure who have exhausted standard of care options. In these emergency situations, Ultragenyx has offered UX07's use. Outcomes have been mixed (as to be expected). Importantly, Ultragenyx has garnered valuable information and prepared a report on their treatment process with UX07, with plans to explore doing a trial specifically for infant cardiomyopathies.

Deflazacort Expanded Access Program for the Treatment of Patients with Duchenne Muscular Dystrophy. Speaker: Tim Cunniff, Pharm.D. Executive Vice-President, Research and Development, Marathon Pharmaceuticals, LLC

Marathon Pharmaceuticals reported their experience with deflazacort in their expanded access program for the treatment of patients with Duchenne Muscular Dystrophy (DMD). DMD is the most common form of DMD, caused by recessive $\mathrm{X}$-linked mutations most commonly manifesting in boys, resulting in muscular degeneration, difficulty walking, breathing and ultimately death. In the US, DMD $\sim 15,000$ boys have the disease and no approved therapies are available.

Deflazacort is a glucocorticoid (steroid) with antiinflammatory, immunosuppressive effects approved in Europe for a wide range of conditions, but not DMD. However, it is used off-label in Europe for the treatment of roughly 1500/2000 DMD patients. While formal studies have not been conducted in these patients, Marathon conducted a 12-week efficacy study on 200 DMD patients comparing deflazacort to prednisone and placebo. The effects of deflazacort compared to prednisone have also been assessed during a one-year study. The Marathon studies of safety revealed important differences between deflazacort and prednisone. Specifically, fewer metabolic and psychiatric side effects were seen with deflazacort treated DMD patients compared to prednisone. Differences in treatment efficacy were also identified, which led to Marathon creating an expanded access program, which subsequently launched in 2015. 
The goal of Marathon's expanded access program was to provide deflazacort to every DMD patient. We anticipated the challenge of providing access to everywhere and wanted patients to have a positive experience closer to home with less travel. Marathon expanded access program offers drug delivery to the patient's home within 24 hours of the placement of the order. Patient outreach is a priority as patients regularly reach out to them if their physicians are not available. To expand the capacity to support expanded access patients, Marathon is also working with advocacy groups through social media to ensure the program is patient friendly.

The key challenges faced included the need of the patients for an investigator's time and the false expectations of what an expanded access program could provide. Specifically, Marathon found itself in a position that they had to explain and reiterate that the deflazacort program was indeed not a clinical trial. To assist communication with healthcare providers, Marathon created an online portal where they could complete the clinical trial agreements, regulatory documents, good laboratory practices (GLPs), order drug, and reports. Marathon is currently collecting data on deflazacort adverse events. Since starting in December of 2015 , the program is now interfacing with about 20 new patients per week.

Industry Case Study: Expanded Access at Achillion Pharmaceuticals. Speaker: Jodie Sherman Gillon, Senior Director, Advocacy \& Professional Society Affairs, Achillion Pharmaceuticals, Inc.

Achillion's is a science-driven, patient-focused company leveraging its strengths across the continuum from drug discovery to commercialization to provide better treatments for people with rare diseases. Several ethical issues have arisen in creating an early access policy and implementing a process. The six main challenges of early access have been: 1) Deciding if your company should provide early access; 2) Determining when expanded access should be implemented; 3) Identifying the preferred approach? (i.e. Multi-patient or individual); 4) Geographical issues; 5) Communication challenges; 6) Financial considerations, and 7) Identifying ways companies improve this process for the stakeholder. In general, these challenges look very different in a large company, where there is broader infrastructure to implement early access, compared to smaller companies.

Early access programs typically start after Phase II or Phase III trials, a time when companies tend to rely on reimbursement. Reimbursement policies vary country to country, with reimbursements taking a considerable amount of time in many parts of the world (it's not such an issue in the United States). This issue is particularly evident in international companies, where these differences can be a burden. This factor is especially important if a patient is receiving benefit from a drug as they will not be removed from the study if they have no other mechanism to get it. However, in doing so, companies take on this financial burden, in which reimbursement could take years to process.

Different countries have different ways of handling expanded access. In some countries, patients are primarily self-pay, so the responsibility of paying for expanded access drugs falls on the patient. In other countries, the government covers the cost of expanded access drugs. In other countries like Brazil, companies are beholden to patients for life, with no guarantee of reimbursement. When considering expanded access on a global scale, the deportation of a drug could be risky because companies may be viewed as promoting an off-label drug. Questions may also be raised regarding why you're not pursuing registration in those countries. There are several ways to mitigate these risks, including clearly stating that your company is providing this service in a reactive fashion, never in a perspective fashion. In that regard, companies should consider having a third pharmacy option as an intermediate between the companies and clinicians and a rationale for not seeking registration in a particular country should be documented.

When communicating with patients, no perfect methods of denying patients early access exist. Achillion has implemented a communication mechanism that involves physician to physician conversations, where the company medical director communicates decisions directly to the requesting physician by phone and email. Other strategies they have found useful include blinding requests for access and responding to requests promptly. Acknowledging requests within a committed time frame (e.g. within five days or 24 hours for emergency situations), for example, is typically expected. If a company is unable to provide access, it is recommended that more than a denial is provided to the patient, including providing other options (i.e. refer a competitor's trial/product, ongoing clinical trial).

Perspectives on Pre-Approval Access to Experimental Medicines: A Case Study. Speaker: Kenneth I. Moch, Managing Partner, The Salutramed Group, LLC

In September of 2009, a request was made to the drug company Chimerix for the drug brincidofovir, an experimental oral anti-viral medication used to treat cytomegalovirus, adenovirus, smallpox, and ebolavirus infections. Over a nine-month period, Chimerix received 50 requests from critically ill patients requesting access to the drug. The FDA requested that Chimerix open an early access program for brincidofovir to help alleviate their burden of approving so many single requests. 
Subsequently, the brincidofovir expanded access program was created. Approximately 400 patients received brincidofovir on a pre-approved access. Upon completion of the study, Chimerix was prepared to terminate the preapproval access program for brincidofovir to focus more on getting the drug approved. Despite this, requests for the drug continued. Over the next 16 months, over 300 requests came into Chimerix for brincidofovir for all different viruses, with the majority of requests coming from individuals, not major medical institutions.

One of the more challenging cases for Chimerix involved a nine-month old that had developed a malignant kidney cancer. Over the course of his life, he survived bouts of four different types of cancer. As a consequence of multiple rounds and types of chemotherapy, he required a bone marrow transplant at the age of 7 , which he received at St. Jude's Hospital in January 2014. In February, 2014 he developed an adenovirus infection, which is associated with a very high mortality in immunocompromised patients (as he was). The physicians at St. Jude's sent a request to Chimerix despite all their other requests having been denied for more than a year. While waiting for the response, his healthcare providers put him on an alternative drug, which happened to be the nephrotoxic parent compound of brincidofovir. As the parent drug began to show nephrotoxicity, St. Jude's made a second request in March of 2014 to Chimerix for brincidofovir. At that time, Chimerix turned down that request. On March 6, 2014, his mother posted on Facebook how ill her son was, which brincidofovir could potentially be used to treat him. At the time, brincidofovir was in Phase III trials in adults for adenovirus. The key rationale for not making brincidofovir available outside of controlled clinical trials was due to the potential of it slowing down or derailing Chimerix abilities to get the drug approved for public-wide access and benefit.

The next morning Chimerix received hundreds of emails and phone calls asking for brincidofovir. Articles were published across the media spectrum on the topic, which did not reflect highly on Chimerix. After this intense social media campaign, the company reversed their decision and released brincidofovir. On March 12, 2014, Chimerix announced the initiation of a pilot Phase III clinical trial, with the 7-year-old that lead to this story as the first patient enrolled. While he did not technically receive brincidofovir under expanded access, their requests through expanded access were the first step to their gaining access. More guidance and framework around a company's role in expanded access are needed, as well as improved mechanisms to assist patients in understanding the regulatory process are needed.

Emerging Gene Therapies. Speaker: Lynne F. McGrath, MPH, PhD. Vice-President of Regulatory Affairs, RegenXBio, Inc.
RegenXbio is an adeno-associated virus (AAV) gene therapy company developing life-altering treatments for patients with severe diseases. The general concept of RegenXbio's gene therapy program is the use of genetically modified AAV to deliver increased levels of a healthy gene. The healthy gene is harbored within cells in addition to the defective gene, to produce an effective product that reverses and/or slows the disease process.

When RegenXbio examined expanded access from a gene therapy view point, several issues were considered. First, AAV gene therapy is a single treatment, providing continued expression of the gene (i.e. treatment) for many years, in contrast to standard therapies with limited coverage. However, what remains unresolved in AAV gene therapy is the issue of knowing how long the gene of interest will continue to express. There are promising data in some experimental models that suggest that AAV-delivered gene expression lasts up to ten years. What this means for early access to investigational gene therapies, however, remains unclear without previous experience or guidance in any similar therapeutic interventions. The issue of persistent expression is also important in developing standard clinical trials.

A second challenge is exactly the opposite problem. Once a gene therapy is given, they cannot be re-dosed. So if sub-optimal levels of gene expression are seen, the resulting lack of response cannot be fully interpreted, in contrast to other drugs where multiple doses may be utilized for effectiveness, particularly in early Phase clinical trials. Another challenge of clinical trials for gene therapy involves inclusion criteria. In some diseases, the optimal time to deliver gene therapy may be early in the disease process so that it will persist through different phases. In other clinical scenarios, it may be more beneficial to deliver the gene later so that it may be more effective later on (keeping in mind the inability to re-dose gene therapies).

The recruitment of patients with orphan diseases is limited to start with and involves companies interfacing with patient advocates to find potential candidates. On top of this limiting factor, another consideration in AAV gene therapy clinical trials involves the exclusion of patients with neutralizing antibodies. A subset of all people have previous exposure to AAV infections and have developed antibodies to clear them, which essentially immunizes these patients against future AAV viruses (including AAV gene therapies). Therefore, all potential AAV gene therapy patients undergo testing for neutralizing antibodies and are excluded if they are found. Lastly, patients who eventually receive gene therapies are monitored for up to 15 years. Since patients with orphan diseases often do not live this long, they may not be as highly considered. These issues contribute to the lack of gene therapies currently approved. 
If expanded access for gene therapies were available, best practices to ensure their successful follow-up over a 15-year period are unclear. Questions remain at how to effectively put gene therapy patients into a registry or group them into clinical trial data. The best access may be to supply an approved therapy.

Janssen Pharmaceuticals Industry Case Study. Speaker: Amrit Ray, MD, MBA. Chief Medical Officer, Janssen Pharmaceuticals, Johnson \& Johnson Pharmaceuticals

At Janssen Pharmaceuticals, all pre-approval access cases have to be done on a foundation of clear pre-identified bioethical principles. Moreover, the application of those principles must be undertaken by independent, objective, and unbiased groups. Therefore, common guidelines are essential to ensure that patients are facing standardized policies across different companies based on disease. At J\&J, all clinical trial data is transparent, and mechanisms for compassionate use are in place, driven by a commitment to ethics.

Over the last three decades, J\&J (Janssen's parent company) has provided early access for its various therapeutics. The last three years, however, stand out as the most remarkable, due in part to the rise in the number of expanded access requests from child patients, social media and the significant steps in legislation and policy.

J\&J manages expanded access in unique ways. The company utilizes forums and has put into place several policies throughout the company with respect to expanded access. These strategies are deeply rooted in J\&J's commitment to maintaining superb ethical principles and supplements the efforts of its compassionate use advisory committee (Compac). J\&J realizes that the responsibly of compassionate use does not solely rest upon the FDA, but upon individual companies. J\&J has partnered with NYU to put together a compact centered on best practices in expanded access. Within the compact are six principles the company agreed upon: 1) Beneficence - The intent of drug development is to help patients. The intent is not to maximize revenue, but to make sure to help the maximum number of patients. 2) Quality - Taking into account the influence of wealth, of celebrity, politics on these decisions. 3) Evidence driven - The decision a company makes should constantly be evolving. 4) Patient-focused - Patients want to hear an answer promptly. 5) Transparency - Making sure data is available. Made a point of publishing. 6) Attention Balancing the attention between the needs of many and needs of a few.

The mission of J\&J compassionate use advisory committee (Compac) includes 1) Making sure that there is one doorway for the whole company. One email address and one phone number for the whole company for compassionate use inquiries. 2) Quick assessment of the Medical risk - Clinical safety profile for each patient and drug. 3) Providing recommendations made back to J\&J and using them to close the loop with the patient's physician and discuss the decisions.

In a small pilot study published in the Journal of the American Medical Association (JAMA), J\&J discussed how they managed the ethical challenges of compassionate use. This study eventually became one of the top $5 \%$ of the mostsearched articles in JAMA. The article examined 160 initial early access requests. Of the 160,84 were determined to be inappropriate (e.g. current therapies had not been exhausted, the form was incomplete, the request involved an unacceptable risk). Of the remaining 76 cases, Compac supplied recommendations, which J\&J concurred with uniformly.

\section{Janssen Pharmaceuticals Industry Case Study from} a Medical Ethicist's Perspective. Alison BatemanHouse, PhD, Assistant Professor, Division of Medical Ethics, NYU School of Medicine

As Janssen Pharmaceuticals was deciphering ethical strategies for managing its expanded access programs, it reached out to the Division of Medical Ethics at New York University (NYU) School of Medicine for assistance. Initially, Bateman-House and Ray formed a working group on compassionate use (Compac). This international academic workgroup (now 25 members) has stakeholders from the FDA, industry, patient advocacy organizations, nurses, and lawyers. When Compac met with J\&J and Janssen to establish a set of ethical principles for the compassionate use of drugs under development, one of the first products they set standards for was daratumumab, a drug used to treat Multiple Myeloma, which was later approved. The Compac would only review patient requests which didn't qualify for a clinical trial or other expanded access programs and had completely exhausted all other treatment options. Since the division of the drug through compassionate use was going to be available internationally, Compac also had to be international, which is why members from five different countries were represented on the committee.

The goal of Compac was the development of consistent, transparent mechanisms for allocating the drug for compassionate use. In this case, daratumumab was in scarce supply. For Compac to make accurate recommendations, constant updates from Janssen on drug availability was constantly needed. The importance of supply was evident once the drug was approved, as completing Compac's mission became much easier once supply was no longer an issue. In the initial stages of daratumumab's development, Compac recommended not allowing access to the drug primarily because there wasn't enough drug to provide.

Multiple Myeloma progression is faster and more severe clinically in certain patient populations, making it necessary for Compac to meet weekly to vote on the expanded access 
cases. Full committee member reviews were made weekly by phone with as many members as possible for a closed member vote. These included physicians, ethicists, and one patient advocate. The Compac took a non-biased approach in accessing daratumumab's efficacy and relied on nonJanssen affiliated advisors for this information. Moreover, multiple myeloma specialists from around the country were hired to join the committee's meetings to help educate them on the drug. To streamline expanded access requests for daratumumab, Compac created standardized forms that interested physicians would fill out, designed in such a way to blind the committee of all personal information about the patient (e.g. where they lived, family history, community involvement, etc.).

A major priority of Compac was first to avoid harm, so if there were any chance of harming the patient, the committee would not give the drug to that patient. Moreover, if the patient was too ill, the committee didn't want to give the drug to that patient, in hopes of supplying it instead to someone that could have a stronger benefit from it. The committee also considered age as a deciding factor in their decision making. One example of this was an emergency decision on expanded access for daratumumab in a 74-year-old man from Israel (at the time Compac was blinded to this information). He had previously participated in a clinical trial with daratumumab and had responded well for some time. However, one of his biomarkers started to increase, and he was removed from the trial. After several months off the drug, his healthcare providers wanted to try the drug again to see if he just needed a reprieve from the drug. The primary mechanism of action that daratumumab works is to target $\mathrm{CD} 38$ overexpressed in multiple myeloma cells. Since this patient had high CD38 levels, the committee agreed that there was a reason to believe that the drug would probably benefit this patient.

MyTomorrows: A platform bridging doctors and patients. Speaker: Ronald Brus, MD, Founder and CEO, myTomorrows

After encountering many hurdles to gaining access to a drug to treat his father's lung carcinoma, Dr. Brus witnessed firsthand the barriers patients face. He also noticed that most approaches to expanded access are drug-centric instead of being patient-centric. What seemed to be lacking were tools to capture real-time data to help counter-balance information asymmetry. Believing that technology is one way to provide solutions in the expanded access space, he developed myTomorrows.

myTomorrows is a platform bridging doctors and patients with new drugs. All countries have expanded access and what the company would like to do for patients and physicians is to unlock the attention of those existing laws. Serving those patients who can't get into clinical trials and automate the most cumbersome processes; most of that being submission to regulatory authorities. myTomorrows ensures that eligibility is reviewed up front, believing that the more data a patient has, the better chance they have at gaining access. The platform also provides web-based navigational tools to match patient demand and doctor's demand for drugs in development. myTomorrows also operates as anonymous brokers to procure the investigational drug for the qualifying patient.

myTomorrows connects patients with drugs by supplying the largest database ever on all clinical trials. For example, the United States-based Clinical trials.gov is only one of 14 , and they want patients to see all trials around the world. myTomorrows aims to structure these trials so anyone can easily find and understand clinical trials related to their disease of interest. The platform gives patients the option to see all early access programs in the world. To address the variability in regulatory standards from country to country, the platform uses geographical sensitive filters to guard against mistakes. For example, when a new drug emerges, the platform provides a course of action to pursue expanded access in a specific region based on geographical regulatory standards. Moreover, the platform is designed to give doctors the right information to help them determine what to prescribe to the patient. Lastly, myTomorrows engages and talks with authorities all over the world to determine reimbursement criteria, in other words, they do not want patients to have to pay for it they want insurance, companies, and governments to pay for it. In return, myTomorrows gives them back the data.

MyTomorrows is active in 16 countries, and about 40,000 patients are on their website every month. The database is updated daily and contains 300,000 trials for about 180 countries, with 40,000-50,000 interventions in different arms of those clinical trials. Moreover, the database contains over 75,000 contact sponsors at some of the major drug companies around the world. A top priority for the company is to ensure compliance will all healthcare laws on a global and national basis. They are making sure that the therapeutics offered for expanded access are of the highest quality. Therefore, the company conducts due diligence on every manufacturer while also monitoring fair pricing and reimbursement.

Session V - The Public Policy Landscape Emerging State \& Federal Laws

The Public Policy Landscape - Emerging State and Federal Laws. Speaker: David Farber, FDA Life Sciences Partner, King \& Spalding Life Sciences \& Healthcare.

The US Congressional Bill HR6270 (Access, Compassion, Care, and Ethics for Seriously Ill Patients Act) is a bipartisan legislation introduced in 2007 led by former Representative Diane E. Watson, a Democrat from California. The bill 
contained a substantial piece on expanded access and worked to establish a procedure for accelerated approval of an investigational drug, biological product, or device that is likely to predict the clinical benefit to a patient suffering from a serious or life-threatening condition. Moreover, it created a much more robust legislative pathway to empower and enable expanded access programs to develop after Phase I trials and provided liability protection to manufacturers.

The following contains a summary of the legislative progress being made with respect to expanded access. Congress (2015 - 2016) Rep. Morgan Griffith (Republican, VA) reintroduces his bill (H.R. 4475) and it fails. Rep. Michael McCaul (Republican, TX) introduces his bill (H.R. 5805), the Andrea Sloan CURE Act (H.R. 6) which is also referred to as the 21st Century CURES bill, which passed the U.S. House of Representatives and exists in pieces within the U.S. Senate, meaning not all sections have been approved. Although the bill did not see action in September of 2016, It is anticipated that many, if not all, sections of the 21st Century CURES bill will be passed in a Congress session reconvening in December of 2016. More recently, the S.2912 bill (Trickett Wendler Right To Try Act of 2016) was introduced by Senator Ron Johnson of Wisconsin. This bill will be very important to follow because it may threaten to cause disruption in the expanded access space.

The CURES Bill (Sections 2081/2082 of H.R.6) outlines the bare essentials of expanded access and constitutes one of the first steps in the public policy debate on expanded access. It requires that pharmaceutical companies that have clinical trials have a website which explains their expanded access policy. For example, it requires companies to provide some criteria as what a company will or will not grant access for. The bill also calls for a clear policy, contact point and information for patients that state how long companies will take to respond. Passage of this bill through congress was expected.

The Right to Try Act will federalize an individual state's right to try an investigational drug, meaning that terminally ill patients within those states will be granted access to investigational drugs that have passed Phase I trials. So far, 31 states have passed "right to try" laws as of September 2016. However, those laws are nullified, because they are preempted by the Food, Drug, and Cosmetic Act. To operationalize the right to try laws, modifications need to be made in the Food, Drug, and Cosmetic Act; and the Right to Try Act will do precisely that. Since being introduced, the bill has garnered very broad-based republican support and two Democrats on the bill. There are 39 health legislative assistants who signed off with their managers in getting on this bill.

Workshop participants were encouraged to take a more active voice in spear heading changes in expanded access policy and legislation. All expanded access constituencies (patient groups) may need a new FDA pathway, meaning there is room to expand or to broaden the statutory limits that the FDA works under today. Understanding what those limits should be and where constituencies need to take them should be the topic of on-going conversations. As stakeholders in the expanded access space, participants were identified as some of the most effective lobbyists on this issue Congress could ever hope for as they have the power to shape that conversation.

\section{Acknowledgements}

The authors wish to thank Ashley G. Rivenbark, PhD for her assistance with editing the manuscript.

\section{References}

1. Meeting detail: http://everylifefoundation.org/8th-annual-scientificworkshop/

2. Organizer/Sponsor: http://everylifefoundation.org/

3. Reagan-Udall Foundation - Expanded Access Patient Navigator http://www.reaganudall.org/wp-content/uploads/2016/04/ Expanded-Access-Navigator-Proposal.pdf

4. Nightingale, Stuart L. "FDA Expanded Access Programs for Experimental Medicines." Jama 314.12 (2015): 1296-1296. http:// jamanetwork.com/journals/jama/fullarticle/2442925

5. Office of Hematology and Oncology Products (OHOP). FDA http:// www.fda.gov/AboutFDA/CentersOffices/OfficeofMedicalProductsandTobacco/CD ER/ucm091745.htm

6. Code of Federal Regulations (21 CFR 312.34) - Treatment use of an investigational new drug https://www.gpo.gov/fdsys/granule/CFR2001-title21-vol5/CFR-2001-title21-vol5-sec312-34

7. Code of Federal Regulations Title 21 (312) - Subpart I--Expanded Access to Investigational Drugs for Treatment Use http://www. accessdata.fda.gov/SCRIPTs/cdrh/cfdocs/cfCFR/CFRSearch. cfm?CFRPart=3 12\&showFR=1\&subpartNode=21:5.0.1.1.3.9

8. American Society of Clinical Oncology (ASCO) University - Expanded Access Courses http://university.asco.org/expanded-access

9. American Cancer Society - Colon Cancer Survival Rates - http://www. cancer.org/cancer/colon-rectal-cancer/detection-diagnosis-staging/ survival-rates.html

10. PhRMA Principles on Conduct of Clinical Trials - http://www.phrma.org/ codes-and-guidelines/phrma-principles-on-conduct-of-clinical-trials

11. The Ethical Challenges of Compassionate Use - http://jamanetwork. com/journals/jama/article-abstract/2491972

12. myTomorrows - https://mytomorrows.com/

13. H.R.6270 - Access, Compassion, Care, and Ethics for Seriously Ill Patients Act - 110th Congress (2007-2008) - https://www.congress. gov/bill/110th-congress/house-bill/6270

14. H.R.6 - 21st Century Cures Act - https://www.congress.gov/ bill/114th-congress/house-bill/6

15. H.R.5805 - Retirement Savings Lost and Found Act of 2016 - 114th Congress (2015-2016) - https://www.congress.gov/bill/114thcongress/house-bill/5805

16. S.2912 - Trickett Wendler Right to Try Act of 2016 - 114th Congress (2015-2016) - https://www.congress.gov/bill/114th-congress/ senate-bill/2912 